\title{
A direct extraction of the Sivers distributions from spin asymmetries in pion and kaon leptoproduction
}

\author{
Anna Martin, ${ }^{1}$ Franco Bradamante,${ }^{2}$ and Vincenzo Barone ${ }^{3}$ \\ ${ }^{1}$ Dipartimento di Fisica, Università degli Studi di Trieste; \\ INFN, Sezione di Trieste, 34127 Trieste, Italy \\ ${ }^{2}$ INFN, Sezione di Trieste, 34127 Trieste, Italy \\ ${ }^{3}$ Di.S.I.T., Università del Piemonte Orientale "A. Avogadro", 15121 Alessandria, Italy; \\ INFN, Sezione di Torino, 10125 Torino, Italy
}

\begin{abstract}
We present a point-by-point determination of the Sivers distributions from hadron leptoproduction data. The method, which relies on some simple assumptions, is based on the combined analysis of proton and deuteron observables. We make use of the single-spin asymmetries measured by COMPASS in semi-inclusive deep inelastic scattering of $160 \mathrm{GeV}$ muons on transversely polarized proton and deuteron targets.

PACS numbers: 13.88.+e, 13.60.-r, 13.66.Bc, 13.85.Ni
\end{abstract}

\section{INTRODUCTION}

One of the most important achievements of hadronic physics in the past decades has been the discovery of significant single-spin asymmetries in leptoproduction of hadrons from a transversely polarized target, $\ell N^{\uparrow} \rightarrow \ell^{\prime} h X$ (for reviews, see e.g. [1]3]). One of these asymmetries is associated with a characteristic angular modulation of the cross section and originates from a correlation between the transverse spin of the nucleon and the transverse momentum of quarks, described by a leading-twist transverse-momentum dependent distribution (TMD), the so-called Sivers function $f_{1 T}^{\perp}[4-7]$.

The Sivers asymmetry has been experimentally observed by the HERMES and COMPASS collaborations in the case of pion and kaon production [8 15]. More recently, data on pion production on a transversely polarized ${ }^{3} \mathrm{He}$ target have been made available by the Hall A Collaboration at JLab [16]. Many phenomenological studies of these measurements are available in the literature [17 27]. In most analyses the Sivers distributions are extracted by fitting the data with a given functional form for the dependence of $f_{1 T}^{\perp}$ on the Bjorken $x$ variable and on the quark intrinsic transverse momentum $k_{T}^{2}$. Here we adopt a different and simpler approach, similar to the one successfully used for the Collins asymmetries in a previous paper of ours [28]. The COMPASS measurements with proton and deuteron targets in the same kinematics allow to perform a point-by-point extraction of the Sivers distributions directly from the data, by properly combining the various asymmetries. Although we use a Gaussian form for the TMD's in order to factorize them from the fragmentation functions, our extraction is essentially parameter-free. In particular, it does not require any specific assumption about the average values of the transverse momenta of quarks. We obtain the Sivers valence distributions both in the case of pion production and in the case of kaon production, and we show that they are compatible with each other.

The plan of the paper is the following. In Sec. II we present the general formalism and write the asymmetries for pion and kaon production, showing that some combinations of them directly provide the Sivers distributions. In Sec. III we extract the valence and sea Sivers distributions from the asymmetries. Finally, Sec. IV contains some concluding remarks.

\section{SIVERS ASYMMETRIES}

\section{A. General formulas}

The process we will be considering is semi-inclusive DIS (SIDIS) with a transversely polarized target, $\ell N^{\uparrow} \rightarrow \ell^{h} h X$. We denote by $\boldsymbol{P}_{h}$ and $M_{h}$ the momentum and the mass, respectively, of the produced hadron. Conventionally, all azimuthal angles are referred to the lepton scattering plane, in a reference system in which the $z$ axis is the virtual photon direction, while the $x$ axis is directed along the transverse momentum of the outgoing lepton: $\phi_{h}$ is the azimuthal angle of $\boldsymbol{P}_{h}, \phi_{S}$ is the azimuthal angle of the nucleon spin vector $\boldsymbol{S}_{\perp}$. The transverse momenta are defined as follows: $\boldsymbol{k}_{T}$ is the transverse momentum 
of the quark inside the nucleon, $\boldsymbol{p}_{T}$ is the transverse momentum of the hadron with respect to the direction of the fragmenting quark, $\boldsymbol{P}_{h \perp}$ is the measurable transverse momentum of the produced hadron with respect to the $z$ axis.

The Sivers term in the cross section, which couples the distribution $f_{1 T}^{\perp}\left(x, k_{T}^{2}, Q^{2}\right)$ to the transversemomentum dependent unpolarized fragmentation function $D_{1}\left(z, p_{T}^{2}, Q^{2}\right)$, is characterized by a $\sin \left(\phi_{h}-\right.$ $\left.\phi_{S}\right)$ modulation. The corresponding asymmetry is [29, 30]

$$
A_{h}\left(x, z, Q^{2}\right)=\frac{\sum_{a} e_{a}^{2} x \int \mathrm{d}^{2} \boldsymbol{P}_{h \perp} \mathcal{C}\left[\frac{\boldsymbol{P}_{h \perp} \cdot \boldsymbol{k}_{T}}{M P_{h \perp}} f_{1 T}^{\perp} D_{1}\right]}{\sum_{a} e_{a}^{2} x \int \mathrm{d}^{2} \boldsymbol{P}_{h \perp} \mathcal{C}\left[f_{1} D_{1}\right]},
$$

where the convolution $\mathcal{C}$ is defined as

$$
\begin{aligned}
\mathcal{C}[w f D]= & \int \mathrm{d}^{2} \boldsymbol{k}_{T} \int \mathrm{d}^{2} \boldsymbol{p}_{T} \delta^{2}\left(z \boldsymbol{k}_{T}+\boldsymbol{p}_{T}-\boldsymbol{P}_{h \perp}\right) \\
& \times w\left(\boldsymbol{k}_{T}, \boldsymbol{p}_{T}\right) f^{a}\left(x, k_{T}^{2}, Q^{2}\right) D^{a}\left(z, p_{T}^{2}, Q^{2}\right) .
\end{aligned}
$$

If we adopt a Gaussian model for the transverse-momentum dependent distribution and fragmentation functions:

$$
\begin{aligned}
& f_{1}\left(x, k_{T}^{2}, Q^{2}\right)=f_{1}\left(x, Q^{2}\right) \frac{\mathrm{e}^{-k_{T}^{2} /\left\langle k_{T}^{2}\right\rangle}}{\pi\left\langle k_{T}^{2}\right\rangle}, \\
& f_{1 T}^{\perp}\left(x, k_{T}^{2}, Q^{2}\right)=f_{1 T}^{\perp}\left(x, Q^{2}\right) \frac{\mathrm{e}^{-k_{T}^{2} /\left\langle k_{T}^{2}\right\rangle_{S}}}{\pi\left\langle k_{T}^{2}\right\rangle_{S}}, \\
& D_{1}\left(z, p_{T}^{2}, Q^{2}\right)=D_{1}\left(z, Q^{2}\right) \frac{\mathrm{e}^{-p_{T}^{2} /\left\langle p_{T}^{2}\right\rangle}}{\pi\left\langle p_{T}^{2}\right\rangle},
\end{aligned}
$$

the Sivers asymmetry (11) takes the form [17, 18, 30]

$$
A_{h}\left(x, z, Q^{2}\right)=G \frac{\sum_{q, \bar{q}} e_{q}^{2} x f_{1 T}^{\perp(1) q}\left(x, Q^{2}\right) z D_{1 q}\left(z, Q^{2}\right)}{\sum_{q, \bar{q}} e_{q}^{2} x f_{1}^{q}\left(x, Q^{2}\right) D_{1 q}\left(z, Q^{2}\right)} .
$$

Here the first $k_{T}^{2}$ moment of the Sivers function is defined as

$$
f_{1 T}^{\perp(1)}\left(x, Q^{2}\right) \equiv \int \mathrm{d}^{2} \boldsymbol{k}_{T} \frac{k_{T}^{2}}{2 M^{2}} f_{1 T}^{\perp}\left(x, k_{T}^{2}, Q^{2}\right),
$$

and $D_{1}\left(z, Q^{2}\right)$ is the fragmentation function integrated over the transverse momentum. The $G$ factor, resulting from the Gaussian integrations, is given by [17, 18]

$$
G=\frac{\sqrt{\pi} M}{\sqrt{\left\langle p_{T}^{2}\right\rangle+z^{2}\left\langle k_{T}^{2}\right\rangle_{S}}}
$$

where $\left\langle k_{T}^{2}\right\rangle_{S}$ is the width of the Sivers distribution. In the Gaussian model the average transverse momentum of the produced hadrons is

$$
\left\langle P_{h \perp}\right\rangle=\frac{\sqrt{\pi}}{2} \sqrt{\left\langle p_{T}^{2}\right\rangle+z^{2}\left\langle k_{T}^{2}\right\rangle},
$$

where $\left\langle k_{T}^{2}\right\rangle$ is the width of the unpolarized $f_{1}$ distribution. The positivity bound for the Sivers function implies that $\left\langle k_{T}^{2}\right\rangle_{S}$ must be smaller than $\left\langle k_{T}^{2}\right\rangle$, but with an error which is well within the overall (experimental + model) uncertainties, we can identify $G$ with

$$
G \simeq \frac{\pi M}{2\left\langle P_{h \perp}\right\rangle} .
$$

$\left\langle P_{h \perp}\right\rangle$ is experimentally found to have a very mild dependence on $x$ and $z$. For simplicity we take its value averaged over $z$, so that the $G$ factor used in our calculations will slightly depend on $x$ only. 
Since our aim is to extract the $k_{T}^{2}$ moment of the Sivers distribution, we integrate over $z$

$$
\widetilde{D}_{1}\left(Q^{2}\right)=\int \mathrm{d} z D_{1}\left(z, Q^{2}\right), \quad \widetilde{D}_{1}^{(1)}\left(Q^{2}\right)=\int \mathrm{d} z z D_{1}\left(z, Q^{2}\right),
$$

and consider the integrated asymmetry:

$$
A_{h}\left(x, Q^{2}\right)=G \frac{\sum_{q, \bar{q}} e_{q}^{2} x f_{1 T}^{\perp(1) q}\left(x, Q^{2}\right) \widetilde{D}_{1 q}^{(1)}\left(Q^{2}\right)}{\sum_{q, \bar{q}} e_{q}^{2} x f_{1}^{q}\left(x, Q^{2}\right) \widetilde{D}_{1 q}\left(Q^{2}\right)} .
$$

\section{B. Pion production}

It is convenient to distinguish favored and unfavored fragmentation functions. For pions they are defined as

$$
\begin{aligned}
& D_{1, \mathrm{fav}}^{\pi} \equiv D_{1 u}^{\pi^{+}}=D_{1 d}^{\pi^{-}}=D_{1 \bar{u}}^{\pi^{-}}=D_{1 \bar{d}}^{\pi^{+}} \\
& D_{1, \mathrm{unf}}^{\pi} \equiv D_{1 u}^{\pi^{-}}=D_{1 d}^{\pi^{+}}=D_{1 \bar{u}}^{\pi^{+}}=D_{1 \bar{d}}^{\pi^{-}} .
\end{aligned}
$$

As for the strange sector, we take

$$
D_{1 s}^{\pi^{ \pm}}=D_{1 \bar{s}}^{\pi^{ \pm}}=N D_{1, \text { unf }}^{\pi}
$$

where $N$ is a constant coefficient. In the fragmentation function parametrization of Ref. [31], $N$ is found to be 0.83 .

The denominators of the asymmetries $\sum_{q, \bar{q}} e_{q}^{2} x f_{1}^{q} \widetilde{D}_{1 q}$, for a proton and a deuteron target $(p, d)$ and for charged pions, multiplied by 9 , are given by (we ignore the charm components of the distribution functions, which are negligible in the kinematic region we are interested in)

$$
\begin{array}{ll}
p, \pi^{+}: & x\left[4\left(f_{1}^{u}+\beta_{\pi} f_{1}^{\bar{u}}\right)+\left(\beta_{\pi} f_{1}^{d}+f_{1}^{\bar{d}}\right)+N \beta_{\pi}\left(f_{1}^{s}+f_{1}^{\bar{s}}\right)\right] \widetilde{D}_{1, \mathrm{fav}}^{\pi} \equiv x f_{p}^{\pi^{+}} \widetilde{D}_{1, \mathrm{fav}}^{\pi}, \\
d, \pi^{+}: & x\left[\left(4+\beta_{\pi}\right)\left(f_{1}^{u}+f_{1}^{d}\right)+\left(1+4 \beta_{\pi}\right)\left(f_{1}^{\bar{u}}+f_{1}^{\bar{d}}\right)+2 N \beta_{\pi}\left(f_{1}^{s}+f_{1}^{\bar{s}}\right)\right] \widetilde{D}_{1, \mathrm{fav}}^{\pi} \equiv x f_{d}^{\pi^{+}} \widetilde{D}_{1, \mathrm{fav}}^{\pi}, \\
p, \pi^{-}: & x\left[4\left(\beta_{\pi} f_{1}^{u}+f_{1}^{\bar{u}}\right)+\left(f_{1}^{d}+\beta_{\pi} f_{1}^{\bar{d}}\right)+N \beta_{\pi}\left(f_{1}^{s}+f_{1}^{\bar{s}}\right)\right] \widetilde{D}_{1, \mathrm{fav}}^{\pi} \equiv x f_{p}^{\pi^{-}} \widetilde{D}_{1, \mathrm{fav}}^{\pi}, \\
d, \pi^{-}: & x\left[\left(1+4 \beta_{\pi}\right)\left(f_{1}^{u}+f_{1}^{d}\right)+\left(4+\beta_{\pi}\right)\left(f_{1}^{\bar{u}}+f_{1}^{\bar{d}}\right)+2 N \beta_{\pi}\left(f_{1}^{s}+f_{1}^{\bar{s}}\right)\right] \widetilde{D}_{1, \mathrm{fav}}^{\pi} \equiv x f_{d}^{\pi^{-}} \widetilde{D}_{1, \mathrm{fav}}^{\pi},
\end{array}
$$

with

$$
\beta_{\pi}\left(Q^{2}\right)=\frac{\widetilde{D}_{1, \mathrm{unf}}^{\pi}\left(Q^{2}\right)}{\widetilde{D}_{1, \mathrm{fav}}^{\pi}\left(Q^{2}\right)} .
$$

Similar expressions can be written for the numerator of Eq. (12), $\sum_{q, \bar{q}} e_{q}^{2} x f_{1 T}^{\perp(1) q} \widetilde{D}_{1 q}^{(1)}$, with the replacements $\widetilde{D}_{1} \rightarrow \widetilde{D}_{1}^{(1)}, f_{1} \rightarrow f_{1 T}^{\perp(1)}$, and $\beta_{\pi} \rightarrow \beta_{\pi}^{(1)}$, where

$$
\beta_{\pi}^{(1)}\left(Q^{2}\right)=\frac{\widetilde{D}_{1, \mathrm{unf}}^{\pi(1)}\left(Q^{2}\right)}{\widetilde{D}_{1, \mathrm{fav}}^{\pi(1)}\left(Q^{2}\right)} .
$$

Introducing the ratio of the first to the zeroth moment of the fragmentation functions,

$$
\rho_{\pi}\left(Q^{2}\right)=\frac{\widetilde{D}_{1, \mathrm{fav}}^{\pi(1)}\left(Q^{2}\right)}{\widetilde{D}_{1, \mathrm{fav}}^{\pi}\left(Q^{2}\right)},
$$

we find for the pion asymmetries with a proton target

$$
\begin{aligned}
& A_{p}^{\pi^{+}}=G \rho_{\pi} \frac{4\left(f_{1 T}^{\perp(1) u}+\beta_{\pi}^{(1)} f_{1 T}^{\perp(1) \bar{u}}\right)+\left(\beta_{\pi}^{(1)} f_{1 T}^{\perp(1) d}+f_{1 T}^{\perp(1) \bar{d}}\right)+N \beta_{\pi}^{(1)}\left(f_{1 T}^{\perp(1) s}+f_{1 T}^{\perp(1) \bar{s}}\right)}{f_{p}^{\pi^{+}}}, \\
& A_{p}^{\pi^{-}}=G \rho_{\pi} \frac{4\left(\beta_{\pi}^{(1)} f_{1 T}^{\perp(1) u}+f_{1 T}^{\perp(1) \bar{u}}\right)+\left(f_{1 T}^{\perp(1) d}+\beta_{\pi}^{(1)} f_{1 T}^{\perp(1) \bar{d}}\right)+N \beta_{\pi}^{(1)}\left(f_{1 T}^{\perp(1) s}+f_{1 T}^{\perp(1) \bar{s}}\right)}{f_{p}^{\pi^{-}}}
\end{aligned}
$$


and for the deuteron target

$$
\begin{aligned}
& A_{d}^{\pi^{+}}=G \rho_{\pi} \frac{\left(4+\beta_{\pi}^{(1)}\right)\left(f_{1 T}^{\perp(1) u}+f_{1 T}^{\perp(1) d}\right)+\left(1+4 \beta_{\pi}^{(1)}\right)\left(f_{1 T}^{\perp(1) \bar{u}}+f_{1 T}^{\perp(1) \bar{d}}\right)+2 N \beta_{\pi}^{(1)}\left(f_{1 T}^{\perp(1) s}+f_{1 T}^{\perp(1) \bar{s}}\right)_{25}}{f_{d}^{\pi^{+}}} \\
& A_{d}^{\pi^{-}}=G \rho_{\pi} \frac{\left(1+4 \beta_{\pi}^{(1)}\right)\left(f_{1 T}^{\perp(1) u}+f_{1 T}^{\perp(1) d}\right)+\left(4+\beta_{\pi}^{(1)}\right)\left(f_{1 T}^{\perp(1) \bar{u}}+f_{1 T}^{\perp(1) \bar{d}}\right)+2 N \beta_{\pi}^{(1)}\left(f_{1 T}^{\perp(1) s}+f_{1 T}^{\perp(1) \bar{s}}\right)_{26)}}{f_{d}^{\pi^{-}}}
\end{aligned}
$$

The combinations

$$
\begin{aligned}
& f_{p}^{\pi^{+}} A_{p}^{\pi^{+}}-f_{p}^{\pi^{-}} A_{p}^{\pi^{-}}=G \rho_{\pi}\left(1-\beta_{\pi}^{(1)}\right)\left(4 f_{1 T}^{\perp(1) u_{v}}-f_{1 T}^{\perp(1) d_{v}}\right) \\
& f_{d}^{\pi^{+}} A_{d}^{\pi^{+}}-f_{d}^{\pi^{-}} A_{d}^{\pi^{-}}=3 G \rho_{\pi}\left(1-\beta_{\pi}^{(1)}\right)\left(f_{1 T}^{\perp(1) u_{v}}+f_{1 T}^{\perp(1) d_{v}}\right)
\end{aligned}
$$

select the valence Sivers distributions. From eqs. (27, 28), we get the valence distributions for $u$ and $d$ quarks separately:

$$
\begin{aligned}
& x f_{1 T}^{\perp(1) u_{v}}=\frac{1}{5 G \rho_{\pi}\left(1-\beta_{\pi}^{(1)}\right)}\left[\left(x f_{p}^{\pi^{+}} A_{p}^{\pi^{+}}-x f_{p}^{\pi^{-}} A_{p}^{\pi^{-}}\right)+\frac{1}{3}\left(x f_{d}^{\pi^{+}} A_{d}^{\pi^{+}}-x f_{d}^{\pi^{-}} A_{d}^{\pi^{-}}\right)\right], \\
& x f_{1 T}^{\perp(1) d_{v}}=\frac{1}{5 G \rho_{\pi}\left(1-\beta_{\pi}^{(1)}\right)}\left[\frac{4}{3}\left(x f_{d}^{\pi^{+}} A_{d}^{\pi^{+}}-x f_{d}^{\pi^{-}} A_{d}^{\pi^{-}}\right)-\left(x f_{p}^{\pi^{+}} A_{p}^{\pi^{+}}-x f_{p}^{\pi^{-}} A_{p}^{\pi^{-}}\right)\right] .
\end{aligned}
$$

A particular combination of proton and deuteron asymmetries selects the sea component of the Sivers function, namely

$$
\begin{aligned}
x f_{1 T}^{\perp(1) \bar{u}}-x f_{1 T}^{\perp(1) \bar{d}}= & \frac{1}{15 G \rho_{\pi}\left(1-\beta_{\pi}^{(1) 2}\right)}\left[2\left(1-4 \beta_{\pi}^{(1)}\right) x f_{p}^{\pi^{+}} A_{p}^{\pi^{+}}+2\left(4-\beta_{\pi}^{(1)}\right) x f_{p}^{\pi^{-}} A_{p}^{\pi^{-}}\right. \\
& \left.\left.-\left(1-4 \beta_{\pi}^{(1)}\right) x f_{d}^{\pi^{+}} A_{d}^{\pi^{+}}-\left(4-\beta_{\pi}^{(1)}\right) x f_{d}^{\pi^{-}} A_{d}^{\pi^{-}}\right)\right] .
\end{aligned}
$$

In Sec. III we will apply Eqs. (29, 30, 31) to extract the Sivers valence and sea distributions.

\section{Kaon production}

In the case of kaons we have two favored fragmentation functions, which largely differ from each other:

$$
\begin{aligned}
& D_{1, \mathrm{fav}}^{K} \equiv D_{1 u}^{K^{+}}=D_{1 \bar{u}}^{K^{-}} \\
& D_{1, \mathrm{fav}}^{\prime K} \equiv D_{1 \bar{s}}^{K^{+}}=D_{1 s}^{K^{-}}
\end{aligned}
$$

Since it is more difficult to excite from the vacuum a heavy $s \bar{s}$ pair than a light $u \bar{u}$ pair, $D_{1, \text { fav }}^{\prime K}$ is expected to be (and in fact is) much larger than $D_{1, \text { fav }}^{K}$. The unfavored fragmentation functions are defined as usual:

$$
D_{1, \mathrm{unf}}^{K} \equiv D_{1 d}^{K^{ \pm}}=D_{1 \bar{d}}^{K^{ \pm}}=D_{1 \bar{u}}^{K^{+}}=D_{1 u}^{K^{-}}=D_{1 s}^{K^{+}}=D_{1 \bar{s}}^{K^{-}}
$$

Proceeding as before, the denominators of the asymmetries $\sum_{q, \bar{q}} e_{q}^{2} x f_{1}^{q} \widetilde{D}_{1 q}$, for kaon production from a proton and a deuteron target $(p, d)$, multiplied by 9 , are given by

$$
\begin{array}{lll}
p, K^{+}: & x\left[4\left(f_{1}^{u}+\beta_{K} f_{1}^{\bar{u}}\right)+\beta_{K}\left(f_{1}^{d}+f_{1}^{\bar{d}}\right)+\left(\beta_{K} f_{1}^{s}+\gamma_{K} f_{1}^{\bar{s}}\right)\right] \widetilde{D}_{1, \mathrm{fav}}^{K} \equiv x f_{p}^{K^{+}} \widetilde{D}_{1, \mathrm{fav}}^{K}, \\
d, K^{+}: & x\left[\left(4+\beta_{K}\right)\left(f_{1}^{u}+f_{1}^{d}\right)+5 \beta_{K}\left(f_{1}^{\bar{u}}+f_{1}^{\bar{d}}\right)+2\left(\beta_{K} f_{1}^{s}+\gamma_{K} f_{1}^{\bar{s}}\right)\right] \widetilde{D}_{1, \mathrm{fav}}^{K} \equiv x f_{d}^{K^{+}} \widetilde{D}_{1, \mathrm{fav}}^{K}, \\
p, K^{-}: & x\left[4\left(\beta_{K} f_{1}^{u}+f_{1}^{\bar{u}}\right)+\beta_{K}\left(f_{1}^{d}+f_{1}^{\bar{d}}\right)+\left(\gamma_{K} f_{1}^{s}+\beta_{K} f_{1}^{\bar{s}}\right)\right] \widetilde{D}_{1, \mathrm{fav}}^{K} \equiv x f_{p}^{K^{-}} \widetilde{D}_{1, \mathrm{fav}}^{K}, \\
d, K^{-}: & x\left[5 \beta_{K}\left(f_{1}^{u}+f_{1}^{d}\right)+\left(4+\beta_{K}\right)\left(f_{1}^{\bar{u}}+f_{1}^{\bar{d}}\right)+2\left(\gamma_{K} f_{1}^{s}+\beta_{K} f_{1}^{\bar{s}}\right)\right] \widetilde{D}_{1, \mathrm{fav}}^{K} \equiv x f_{d}^{K^{-}} \widetilde{D}_{1, \mathrm{fav}}^{K},
\end{array}
$$


where $\beta_{K}$ and $\gamma_{K}$ are defined as

$$
\beta_{K}\left(Q^{2}\right)=\frac{\widetilde{D}_{1, \mathrm{unf}}^{K}\left(Q^{2}\right)}{\widetilde{D}_{1, \mathrm{fav}}^{K}\left(Q^{2}\right)}, \quad \gamma_{K}\left(Q^{2}\right)=\frac{\widetilde{D}_{1, \mathrm{fav}}^{\prime K}\left(Q^{2}\right)}{\widetilde{D}_{1, \mathrm{fav}}^{K}\left(Q^{2}\right)},
$$

For the numerator of Eq. (12), $\sum_{q, \bar{q}} e_{q}^{2} x f_{1 T}^{\perp(1) q} \widetilde{D}_{1 q}^{(1)}$, one can write similar expressions with the replacements $\widetilde{D}_{1} \rightarrow \widetilde{D}_{1}^{(1)}, f_{1} \rightarrow f_{1 T}^{\perp(1)}, \beta_{K} \rightarrow \beta_{K}^{(1)}$ and $\gamma_{K} \rightarrow \gamma_{K}^{(1)}$, where $\beta_{K}^{(1)}$ and $\gamma_{K}^{(1)}$ are given by

$$
\beta_{K}^{(1)}\left(Q^{2}\right)=\frac{\widetilde{D}_{1, \text { unf }}^{K(1)}\left(Q^{2}\right)}{\widetilde{D}_{1, \text { fav }}^{K(1)}\left(Q^{2}\right)}, \quad \gamma_{K}^{(1)}\left(Q^{2}\right)=\frac{\widetilde{D}_{1, \mathrm{fav}}^{\prime K(1)}\left(Q^{2}\right)}{\widetilde{D}_{1, \mathrm{fav}}^{K(1)}\left(Q^{2}\right)},
$$

The resulting $K^{ \pm}$Sivers asymmetries are:

$$
\begin{aligned}
& A_{p}^{K^{+}}=G \rho_{K} \frac{4\left(f_{1 T}^{\perp(1) u}+\beta_{K}^{(1)} f_{1 T}^{\perp(1) \bar{u}}\right)+\beta_{K}^{(1)}\left(f_{1 T}^{\perp(1) d}+f_{1 T}^{\perp(1) \bar{d}}\right)+\left(\beta_{K}^{(1)} f_{1 T}^{\perp(1) s}+\gamma_{K}^{(1)} f_{1 T}^{\perp(1) \bar{s}}\right)}{f_{p}^{K^{+}}}, \\
& A_{p}^{K^{-}}=G \rho_{K} \frac{4\left(\beta_{K}^{(1)} f_{1 T}^{\perp(1) u}+f_{1 T}^{\perp(1) \bar{u}}\right)+\beta_{K}^{(1)}\left(f_{1 T}^{\perp(1) d}+f_{1 T}^{\perp(1) \bar{d}}\right)+\left(\gamma_{K}^{(1)} f_{1 T}^{\perp(1) s}+\beta_{K}^{(1)} f_{1 T}^{\perp(1) \bar{s}}\right)}{f_{p}^{K^{-}}}, \\
& A_{d}^{K^{+}}=G \rho_{K} \frac{\left(4+\beta_{K}^{(1)}\right)\left(f_{1 T}^{\perp(1) u}+f_{1 T}^{\perp(1) d}\right)+5 \beta_{K}^{(1)}\left(f_{1 T}^{\perp(1) \bar{u}}+f_{1 T}^{\perp(1) \bar{d}}\right)+2\left(\beta_{K}^{(1)} f_{1 T}^{\perp(1) s}+\gamma_{K}^{(1)} f_{1 T}^{\perp(1) \bar{s}}\right)}{f_{d}^{K^{+}}}, \\
& A_{d}^{K^{-}}=G \rho_{K} \frac{5 \beta_{K}^{(1)}\left(f_{1 T}^{\perp(1) u}+f_{1 T}^{\perp(1) d}\right)+\left(4+\beta_{K}^{(1)}\right)\left(f_{1 T}^{\perp(1) \bar{u}}+f_{1 T}^{\perp(1) \bar{d}}\right)+2\left(\gamma_{K}^{(1)} f_{1 T}^{\perp(1) s}+\beta_{K}^{(1)} f_{1 T}^{\perp(1) \bar{s}}\right)}{f_{d}^{K^{-}}},
\end{aligned}
$$

with the moment ratio $\rho_{K}$ defined as

$$
\rho_{K}\left(Q^{2}\right)=\frac{\widetilde{D}_{1, \mathrm{fav}}^{K(1)}\left(Q^{2}\right)}{\widetilde{D}_{1, \mathrm{fav}}^{K}\left(Q^{2}\right)} .
$$

By combining the asymmetries, we get

$$
\begin{aligned}
f_{p}^{K^{+}} A_{p}^{K^{+}}-f_{p}^{K^{-}} A_{p}^{K^{-}}=G \rho_{K} & {\left[4\left(1-\beta_{K}^{(1)}\right) f_{1 T}^{\perp(1) u_{v}}+\left(\beta_{K}^{(1)}-\gamma_{K}^{(1)}\right)\left(f_{1 T}^{\perp(1) s}-f_{1 T}^{\perp(1) \bar{s}}\right)\right] } \\
f_{d}^{K^{+}} A_{d}^{K^{+}}-f_{d}^{K^{-}} A_{d}^{K^{-}}=G \rho_{K} & {\left[4\left(1-\beta_{K}^{(1)}\right)\left(f_{1 T}^{\perp(1) u_{v}}+f_{1 T}^{\perp(1) d_{v}}\right)\right.} \\
+ & \left.2\left(\beta_{K}^{(1)}-\gamma_{K}^{(1)}\right)\left(f_{1 T}^{\perp(1) s}-f_{1 T}^{\perp(1) \bar{s}}\right)\right] .
\end{aligned}
$$

In order to extract $u_{V}$ and $d_{V}$ separately, we assume that the difference of strange sea distributions, $s-\bar{s}$, is negligible. Thus we obtain

$$
\begin{aligned}
& x f_{1 T}^{\perp(1) u_{v}}=\frac{1}{4 G \rho_{K}\left(1-\beta_{K}^{(1)}\right)}\left[\left(x f_{p}^{K^{+}} A_{p}^{K^{+}}-x f_{p}^{K^{-}} A_{p}^{K^{-}}\right)\right] \\
& x f_{1 T}^{\perp(1) d_{v}}=\frac{1}{4 G \rho_{K}\left(1-\beta_{K}^{(1)}\right)}\left[\left(x f_{d}^{K^{+}} A_{d}^{K^{+}}-x f_{d}^{K^{-}} A_{d}^{K^{-}}\right)-\left(x f_{p}^{K^{+}} A_{p}^{K^{+}}-x f_{p}^{K^{-}} A_{p}^{K^{-}}\right)\right] .
\end{aligned}
$$

These two relations will be used to extract the valence Sivers distributions from kaon data.

We note that by using the full set of eight asymmetries experimentally measured (proton and deuteron targets, $\pi^{ \pm}$and $K^{ \pm}$productions) one could in principle obtain more information on the distributions, in particular on the strange and non-strange sea, but we prefer to consider only the functions that can be directly determined by linear combinations of the asymmetries. 


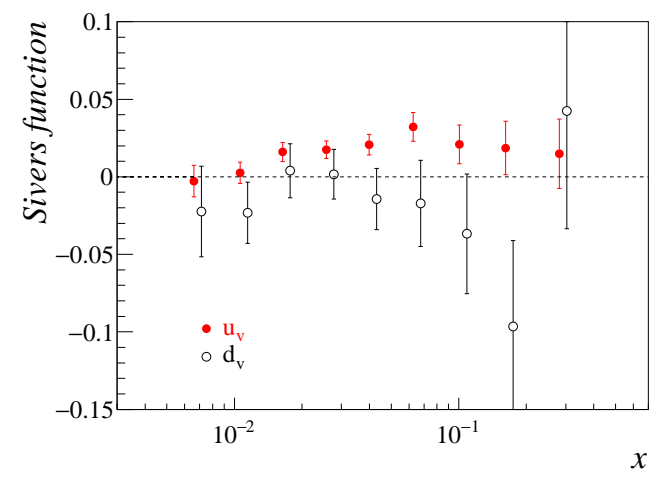

FIG. 1: The first $k_{T}^{2}$ moments of the Sivers valence distributions, $x f_{1 T}^{\perp(1) u_{v}}$ (red solid circles) and $x f_{1 T}^{\perp(1) d_{v}}$ (black open circles), extracted from pion asymmetries.

\section{EXTRACTION OF THE SIVERS DISTRIBUTIONS}

As we have seen, it is possible to obtain directly the valence and the sea components of the Sivers function by combining different asymmetries. The data we use are from COMPASS measurements of semi-inclusive deep inelastic scattering on proton [15] and deuteron targets [12].

In order to extract $f_{1 T}^{\perp(1)}$ we need two extra ingredients: the unpolarized distribution functions $f_{1}^{q}$, which are taken from the CTEQ5D global fit [32], and the unpolarized fragmentation functions, which are taken from the DSS parametrization [31]. Notice that in the DSS fit of pion fragmentation functions $D_{1 u}^{\pi^{+}}$is not assumed to be equal to $D_{1 \bar{d}}^{\pi^{+}}$, but their difference is rather small. Thus, we identify $D_{1, \text { fav }}$ with $\left(D_{1 u}^{\pi^{+}}+D_{1 \bar{d}}^{\pi^{+}}\right) / 2$ as given by DSS.

The normalization of the Sivers distributions is determined by the quantity $G=\pi M / 2\left\langle P_{h \perp}\right\rangle$. The values of $\left\langle P_{h \perp}\right\rangle$, measured by COMPASS, slightly depend on $x$, so that $G$ ranges from 2.8 to 3.1 for pions, and from 2.4 to 2.6 for kaons.

We can now use Eqs. (29, 30) to extract point-by-point the valence Sivers distributions from pion data. The results are tabulated in Table 1 and displayed in Fig. 1. The error bars are computed from the statistical errors of the measured asymmetries, and no attempt has been made to try to assign a systematic error to the results. Notice also that the $x$ points correspond to different $Q^{2}$ values, ranging from $1.2 \mathrm{GeV}^{2}$ to $20 \mathrm{GeV}^{2}$, with an average value $\left\langle Q^{2}\right\rangle \approx 4 \mathrm{GeV}^{2}$.

\begin{tabular}{|c|c|c|c|}
\hline$x$ & $Q^{2}\left(\mathrm{GeV}^{2}\right)$ & $x f_{1 T}^{\perp(1) u_{v}}$ & $x f_{1 T}^{\perp(1) d_{v}}$ \\
\hline 0.007 & 1.22 & $-0.003 \pm 0.010$ & $-0.022 \pm 0.029$ \\
0.011 & 1.43 & $0.003 \pm 0.007$ & $-0.023 \pm 0.020$ \\
0.016 & 1.66 & $0.016 \pm 0.006$ & $0.004 \pm 0.017$ \\
0.026 & 1.96 & $0.017 \pm 0.006$ & $0.002 \pm 0.016$ \\
0.040 & 2.57 & $0.021 \pm 0.007$ & $-0.014 \pm 0.020$ \\
0.063 & 4.01 & $0.032 \pm 0.009$ & $-0.017 \pm 0.028$ \\
0.101 & 6.38 & $0.021 \pm 0.012$ & $-0.037 \pm 0.039$ \\
0.162 & 9.91 & $0.019 \pm 0.017$ & $-0.096 \pm 0.055$ \\
0.281 & 20.23 & $0.015 \pm 0.022$ & $0.042 \pm 0.076$ \\
\hline
\end{tabular}

TABLE I: Sivers valence distributions extracted from pion asymmetries.

The $u_{v}$ distribution is determined much more precisely than the $d_{v}$ distribution, due to the fact that the asymmetry measurements on the proton are considerably more accurate than the corresponding ones on the deuteron, in particular in the valence region (the COMPASS Collaboration has taken much less data on deuterons than on protons). Still, the $d_{v}$ Sivers function is reasonably well determined and turns out to be negative and approximately specular to the $u_{v}$ function.

Equation (31) allows determining directly the isotriplet $\bar{u}-\bar{d}$ component of the Sivers sea. The results 
are shown in Fig. 2 and have errors comparable to those of the $u_{v}$ function. In the large $N_{c}$ limit, the isotriplet $(\bar{u}-\bar{d})$ Sivers combination is expected to dominate over the isosinglet one $(\bar{u}+\bar{d})$ [33], thus the vanishing of $x f_{1 T}^{\perp(1) \bar{u}}-x f_{1 T}^{\perp(1) \bar{d}}$ is not due to a cancellation of the two terms, but rather signals the smallness of the $\bar{u}$ and $\bar{d}$ separately.

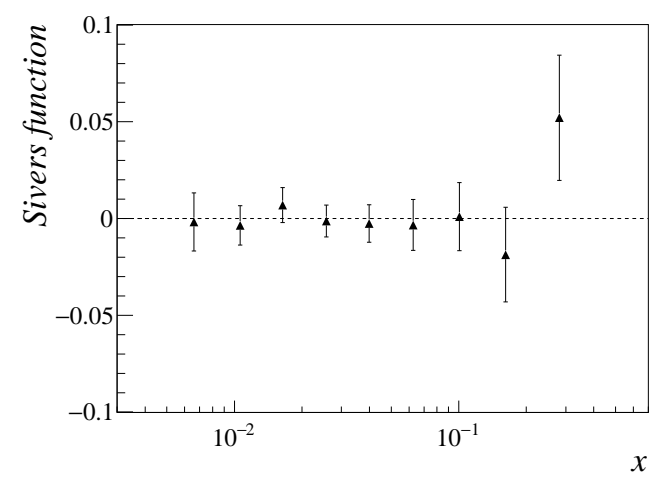

FIG. 2: The isotriplet Sivers sea $x f_{1 T}^{\perp(1) \bar{u}}-x f_{1 T}^{\perp(1) \bar{d}}$ extracted from pion asymmetry data.

A similar procedure has been applied to extract the Sivers functions from the measured kaon asymmetries. The Sivers valence distributions, extracted using Eqs. (48, 49), are shown in Table 1 and Fig. 3 . Notice that the $Q^{2}$ values in each $x$ bin are slightly larger than for pions. Again, the $u_{v}$ distribution is well determined, whereas in this case the $d_{v}$ distribution is affected by large uncertainties and does not exhibit a clear behavior.

\begin{tabular}{|c|c|c|c|}
\hline$x$ & $Q^{2}\left(\mathrm{GeV}^{2}\right)$ & $x f_{1 T}^{\perp(1) u_{v}}$ & $x f_{1 T}^{\perp(1) d_{v}}$ \\
\hline 0.007 & 1.21 & $-0.003 \pm 0.016$ & $0.000 \pm 0.057$ \\
0.011 & 1.43 & $-0.006 \pm 0.010$ & $0.015 \pm 0.036$ \\
0.016 & 1.75 & $0.015 \pm 0.009$ & $0.020 \pm 0.033$ \\
0.026 & 2.31 & $0.011 \pm 0.010$ & $0.046 \pm 0.034$ \\
0.040 & 3.34 & $0.021 \pm 0.012$ & $0.086 \pm 0.043$ \\
0.063 & 5.16 & $0.026 \pm 0.015$ & $-0.015 \pm 0.052$ \\
0.101 & 8.01 & $0.054 \pm 0.018$ & $-0.100 \pm 0.062$ \\
0.162 & 12.78 & $0.060 \pm 0.023$ & $-0.061 \pm 0.078$ \\
0.281 & 26.47 & $0.030 \pm 0.024$ & $-0.028 \pm 0.096$ \\
\hline
\end{tabular}

TABLE II: Sivers valence distributions extracted from kaon production.

The valence Sivers distributions extracted from pion and kaon leptoproduction data are compared in Fig. 4. In the case of $u_{v}$ the two sets of points are compatible with each other, as they should be, representing the same universal property of the target (minor differences in the $Q^{2}$ values of pion and kaon points can be practically ignored). The $d_{v}$ functions are also similar to each other, although affected by much larger errors.

In Fig. 5 we show the weighted averages of the Sivers distributions obtained from pion and kaon data. For comparison we plot the results (central values and uncertainty bands) of the fit of Ref. [25] based on DGLAP evolution. Note that these results refer to $Q^{2}=4 \mathrm{GeV}^{2}$, the average momentum transfer of COMPASS measurements, whereas our points correspond to different $Q^{2}$ values, as explained above. However, except for the first few points at low $x$ and small momentum transfer, the $Q^{2}$ evolution is not expected to affect the results significantly. The TMD evolution [34, 35] has also been applied to the analysis of the Sivers data, but in this scheme the perturbative evolution is driven by a factor which cancels out in the asymmetry ratio. Thus, a fit to the asymmetry data based on the TMD evolution is not able to constrain the absolute normalization of the Sivers distributions (see the discussion in Ref. [25]). 


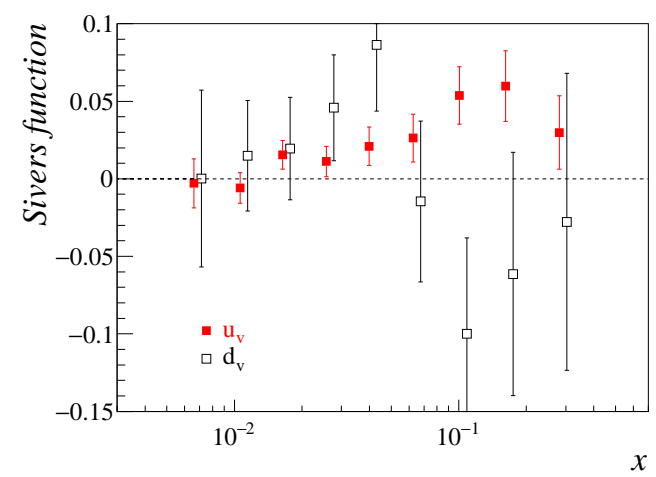

FIG. 3: The first $k_{T}^{2}$ moments of the Sivers valence distributions, $x f_{1 T}^{\perp(1) u_{v}}$ (red solid squares) and $x f_{1 T}^{\perp(1) d_{v}}$ (black open squares), extracted from kaon asymmetries.
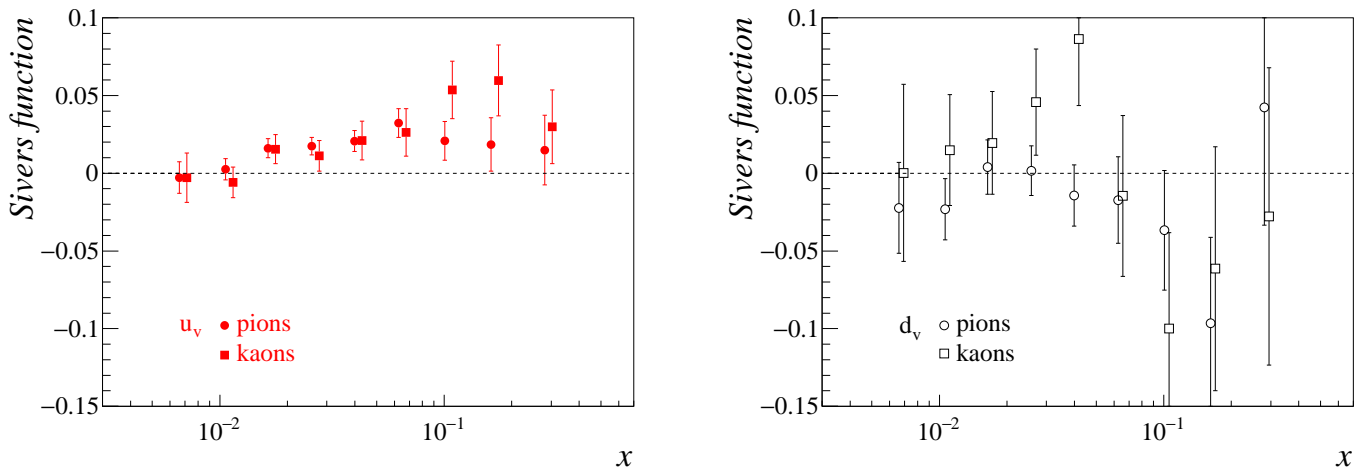

FIG. 4: Comparison of the first $k_{T}^{2}$ moments of the Sivers valence distributions, $x f_{1 T}^{\perp(1) u_{v}}$ (left panel) and $x f_{1 T}^{\perp(1) d_{v}}$ (right panel), obtained from pion and kaon data.

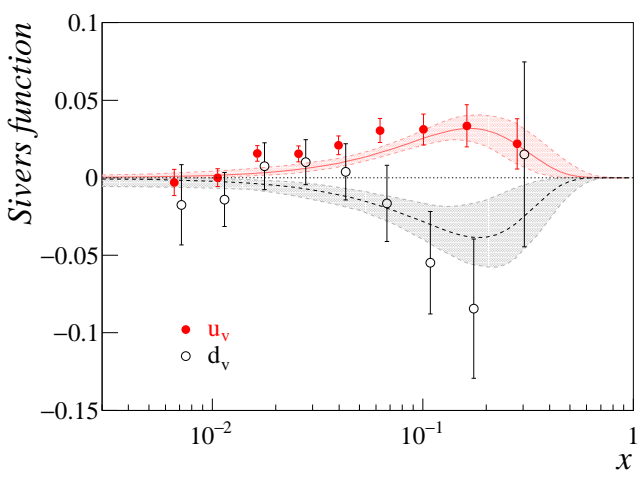

FIG. 5: The average Sivers distributions $x f_{1 T}^{\perp(1) u_{v}}$ (solid points) and $x f_{1 T}^{\perp(1) d_{v}}$ (open points) obtained from pion and kaon data. Our points are compared to the results of the fit of Ref. [25] for $Q^{2}=4 \mathrm{GeV}^{2}$ (central values and uncertainty bands). 


\section{CONCLUDING REMARKS}

In summary, we extracted in a simple and direct way the Sivers distributions from the COMPASS measurements of pion and kaon leptoproduction on proton and deuteron targets. The main assumption we made in order to factorize the Sivers functions from the fragmentation functions was the Gaussian behavior in the transverse momenta. As expected, the $u_{v}$ and $d_{v}$ distributions extracted from the pion data are well compatible with the corresponding ones extracted from the kaon data, and the final results have been obtained by averaging the two partial results. The distributions are roughly mirror-symmetric and of similar magnitude, the $u_{v}$ being positive and the $d_{v}$ negative. They are in good agreement with the results of previous fits which assumed a functional form for the distributions from the very beginning.

While the $u_{v}$ distribution is determined with a satisfactory accuracy, the $d_{v}$ distribution is more uncertain. To improve its knowledge more data are needed, in particular on the deuteron. The long-term solution would be the planned Electron Ion Collider, but in the near future the proposed new COMPASS run on a deuteron target [36] would certainly provide new precious information.

Another interesting result from our work is the extraction of the Sivers sea $\bar{u}-\bar{d}$. This is found to be compatible with zero, but it is interesting to notice that the accuracy of this result is comparable to that of the valence distributions.

We conclude by recalling that the Sivers function can be disentangled from the transverse momentum convolution and extracted in a fully model-independent way (i.e., with no Gaussian assumption) by

considering the asymmetries $A_{h}^{w}$ weighted with $P_{h \perp}$ [30]. The COMPASS Collaboration is currently working on the analysis of the weighted Sivers asymmetries. The method illustrated in the present paper can be applied to those observables in a straightforward way.

\section{Acknowledgments}

We are grateful to M.E. Boglione for providing us the curves of the fits of Ref. [25].

[1] V. Barone, F. Bradamante and A. Martin, Progr. Part. Nucl. Phys. 65, 267 (2010).

[2] C.A. Aidala, S.D. Bass, D. Hasch, and G.K. Mallot, Rev. Mod. Phys. 85, 655 (2013).

[3] The 3-D Structure of the Nucleon, special issue of Eur. Phys. J. A 52, no. 6, 2016.

[4] D. Sivers, Phys. Rev. D 41, 83 (1990).

[5] D. Sivers, Phys. Rev. D 43, 261 (1991).

[6] S.J. Brodsky, D.S. Hwang, and I. Schmidt, Phys. Lett. B 530, 99 (2002).

[7] J.C. Collins, Phys. Lett. B 536, 43 (2002)

[8] A. Airapetian et al. (HERMES Collaboration), Phys. Rev. Lett. 94, 012002 (2005).

[9] V.Yu. Alexakhin et al. (COMPASS Collaboration), Phys. Rev. Lett. 94, 202002 (2005).

[10] E. S. Ageev et al. (COMPASS Collaboration), Nucl. Phys. B 765, 31 (2007).

[11] A. Airapetian et al. (HERMES Collaboration), Phys. Rev. Lett. 103, 152002 (2009).

[12] M. Alekseev et al. (COMPASS Collaboration), Phys. Lett. B 673, 127 (2009).

[13] M. G. Alekseev et al. (COMPASS Collaboration), Phys. Lett. B 692, 240 (2010).

[14] C. Adolph et al. (COMPASS Collaboration), Phys. Lett. B 717, 107 (2012).

[15] C. Adolph et al. (COMPASS Collaboration), Phys. Lett. B 744, 250 (2015).

[16] X. Qian et al. (JLab Hall A Collaboration), Phys. Rev. Lett. 107, 072003 (2011).

[17] A.V. Efremov, K. Goeke, and P. Schweitzer, Phys. Lett. B 568, 63 (2003).

[18] A.V. Efremov, K. Goeke, S. Menzel, A. Metz, and P. Schweitzer, Phys. Lett. B 612, 233 (2005).

[19] J.C. Collins, A.V. Efremov, K. Goeke, S. Menzel, A. Metz, and P. Schweitzer, Phys. Rev. D 73, 014021 (2006).

[20] W. Vogelsang, and F. Yuan, Phys. Rev. D 72, 054028 (2005).

[21] M. Anselmino, M. Boglione, U. D'Alesio, A. Kotzinian, F. Murgia, and A. Prokudin, Phys. Rev. D 71, 074006 (2005).

[22] M. Anselmino, M. Boglione, U. D’Alesio, A. Kotzinian, F. Murgia, and A. Prokudin, Phys. Rev. D 72, 094007 (2005).

[23] M. Anselmino et al., in Transversity 2005, V. Barone and P.G. Ratcliffe, eds., World Scientific, Singapore (2006), p. 236; arXiv:hep-ph/0511017. 
[24] M. Anselmino, M. Boglione, U. D’Alesio, A. Kotzinian, S. Melis, F. Murgia, A. Prokudin, and C. Türk, Eur. Phys. J. A 39, 89 (2008).

[25] M. Anselmino, M. Boglione, and S. Melis, Phys. Rev. D 86, 014028 (2012).

[26] P. Sun and F. Yuan, Phys. Rev. D 88, 034016 (2013).

[27] M.G. Echevarria, A. Idilbi, Z.-B. Kang, and I. Vitev, Phys. Rev. D 89, 074013 (2014).

[28] A. Martin, F. Bradamante, and V. Barone, Phys. Rev. D 91, 014034 (2015).

[29] P.J. Mulders and R.D. Tangerman, Nucl. Phys. B461, 197 (1996).

[30] D. Boer and P.J. Mulders, Phys. Rev. D 57, 5780 (1998).

[31] D. de Florian, R. Sassot and M. Stratmann, Phys. Rev. D 75, 114010 (2007).

[32] H.L. Lai et al. (CTEQ Collaboration), Eur. Phys. J. C12, 375 (2000).

[33] P.V. Pobylitsa, arXiv:hep-ph/0301236

[34] S.M. Aybat and T.C. Rogers, Phys. Rev. D 83, 114042 (2011).

[35] S.M. Aybat, J.C. Collins, J.-W. Qiu, and T.C. Rogers, Phys. Rev. D 85, 034043 (2012).

[36] COMPASS Collaboration, "COMPASS Planned Measurements in the next five years and longer term perspectives on the study of the nucleon structure", July 30, 2012, report for the European Strategy Preparatory Group. 